\title{
Endogenous Level and in Vitro Production of GHB in Blood from Healthy Humans, and the Interpretation of GHB Levels Detected in Antemortem Blood Samples
}

\author{
Noriaki Shima,* Akihiro Miki, Tooru Kamata, Munehiro Katagi, and Hitoshi Tsuchihashi \\ Forensic Science Laboratory, Osaka Prefectural Police Headquarters, 1-3-18 Hommachi, Chuo-ward, Osaka 541-0053, Japan
}

(Received September 25, 2004; Accepted December 22, 2004; Published online January 7, 2005)

\begin{abstract}
The endogenous level of $\gamma$-hydroxybutyric acid (GHB) and the in vitro production of GHB in blood from healthy humans have been investigated. The endogenous GHB concentrations in aseptically collected whole blood samples ranged from 5 to $10 \mathrm{ng} / \mathrm{ml}$, which were far below the previously-reported "endogenous" levels . Also, the levels of in vitro GHB production during storage for 16 months at $4{ }^{\circ} \mathrm{C}$ were lower than $0.4 \mu \mathrm{g} / \mathrm{ml}$, which were much lower than those in postmortem samples previously reported. Based on the results of this investigation, the authors concluded that an interpretative cutoff of $1.0 \mu \mathrm{g} / \mathrm{ml}$ would be appropriate for differentiating exogenous from endogenous GHB, if only limited to in-life blood specimens that were collected aseptically, stored at $4^{\circ} \mathrm{C}$ or lower, examined within two weeks, and excludes the possibility of GHB aciduria.
\end{abstract}

Key words — gamma hydroxybutyric acid, endogenous level, blood, gas chromatography mass spectrometry, forensic toxicology

\section{INTRODUCTION}

Gamma-hydroxybutyric acid (GHB) is a central nervous system depressant, which produces noticeable depressant effects, such as unconsciousness, seizures, severe respiratory depression, and come at high dosages. ${ }^{1,2)}$ In the last decade, GHB has become one of the most frequently-encountered drugs used for both abusive and malicious purposes, such as a club drug and date-rape drug. ${ }^{3)}$ Because of the escalating popularity of GHB, it was temporarily designated as a federally-controlled Schedule I substance in the United States in $2000{ }^{4}{ }^{4}$ though with its recent approval for clinical use, prescription GHB is currently a Schedule III substance. Japan's authorities also banned this substance in 2001 under the Narcotics and Psychotropics Control Law to prevent its spread for recreational and malicious use.

In most mammalians, however, GHB occurs as an endogenous metabolite of GABA ( $\gamma$-aminobutyric

\footnotetext{
*To whom correspondence should be addressed: Forensic Science Laboratory, Osaka Prefectural Police Headquarters, 1-318 Hommachi, Chuo-ward, Osaka 541-0053, Japan. Tel.: +816-6268-1234; Fax: +81-6-6271-8066; E-mail: nori6499@ amber. plala.or.jp
}

acid) at nanomolar concentrations in tissues, ${ }^{5)}$ and thus trace-level GHB is found in many foodstuffs, as well as in human blood. GHB is extensively metabolized into succinic acid via succinic semialdehyde, with resultant production of carbon dioxide and water via the Krebs cycle, ${ }^{6}$ as summarized in Fig. 1. Such endogenous nature and high metabolism of GHB makes the differentiation of exogenous GHB in blood from endogenous one problematic.

Compounding difficulties include the in-vitro production of GHB in blood during sample storage. Several papers reported the "endogenous" GHB levels in blood, but such levels vary widely most probably depending on the sample storage conditions, as well as on the origin of the sample (i.e., postmortem blood or blood freshly collected from a living human). ${ }^{7,8)}$ In fact, notably high levels of GHB (typically around $10 \mu \mathrm{g} / \mathrm{ml}$ or more) were detected in postmortem blood samples of non-GHB cases, while trace-level GHB (typically less than $1.0 \mu \mathrm{g} / \mathrm{ml}$ ) was detectable in freshly collected blood from healthy humans. Also, Elliott demonstrated that manners in sample handling and analytical techniques employed affect on its "endogenous" level in blood. ${ }^{8)}$

Moreover, the non-controlled equivalents or al- 


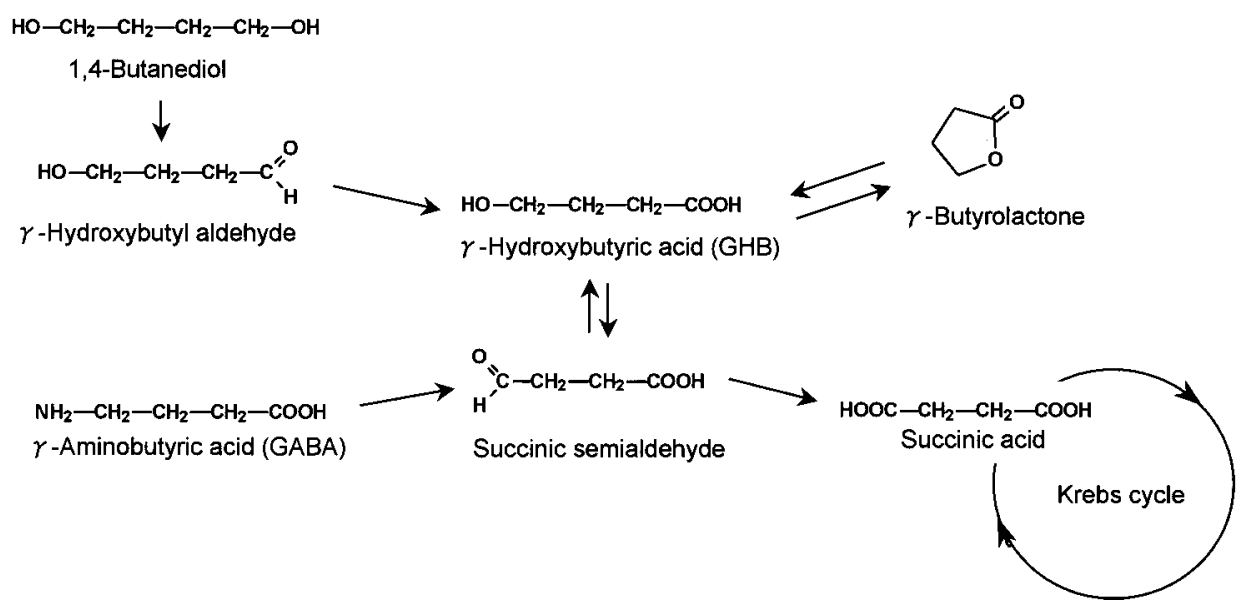

Fig. 1. Biochemical Synthetic and Metabolic Pathways of GHB and its Related Compounds

ternatives to GHB, such as $\gamma$-butyloractone (GBL) and 1,4-butanediol, have gained popularity instead of the recently banned GHB. Since such analogs are biotransformed into GHB in the body and thus act as $\mathrm{GHB},{ }^{9-11)}$ there is an increasing need for the analysis of such exogenous GHB in the instances of drugfacilitated sexual assert (DFSA), driving under the influence of drugs (DUI), and so on. This information indicates further investigation on in-life endogenous blood GHB level, interpretative cutoff settings, as well as preventive measures for the in-vitro production of GHB is required, in order to unequivocally differentiate the intake of GHB or its equivalents from endogenous GHB.

In our previous study, we established a sensitive and reliable GC-MS procedure for the simultaneous determination of GHB and its isomers in urine. ${ }^{12)}$ In the present study, by using the optimized method with modification, endogenous blood GHB level has been investigated in drug-free healthy humans. Based on the research results, the administrative cutoff of blood GHB for proving exogenous GHB in blood from living humans will be investigated. Because in vitro GHB production is often found during sample storage, preventive measures for such GHB production has also been investigated.

\section{MATERIALS AND METHODS}

Reagents — GHB sodium salt was purchased from Sigma Chemical (St. Louis, MO, U.S.A.). $\alpha$ hydroxybutyric acid (AHB) and $\beta$-hydroxybutyric acid (BHB) were obtained from Wako Pure Chemical Industries (Osaka, Japan). 2-Hydroxycaproic acid
(HCA) used as an internal standard (IS) was obtained from Aldrich (Tokyo, Japan). Stock standard solutions of these substances were prepared in methanol, and were diluted with water to appropriate concentrations as needed. $\mathrm{N}, \mathrm{O}$-bis(trimethylsilyl) trifluoroacetamide (BSTFA) with 1\% trimethylchlorosilane (TMCS) was purchased from Spelco (Bellefonte, PA, U.S.A.).

Specimens — Whole blood specimens were anonymously provided and collected from 24 healthy volunteers (14 males and 10 females, aged between 25 and 55 years). All subjects were Asians. The specimens were aseptically collected into vacuum stoppered polyethylene test tubes and were immediately cooled and stored at $-20^{\circ} \mathrm{C}$ without adding any preservatives. The specimens were processed within three hours from sampling and analyzed promptly as described below. Fortified blood samples used for method validation were prepared by adding known amounts of GHB to a healthy volunteer's blood sample immediately prior to the use.

Sample Processing — To $200 \mu \mathrm{l}$ of whole blood samples, $100 \mu \mathrm{l}$ of $0.1 \mu \mathrm{g} / \mathrm{ml}$ aqueous solution of the IS was mixed, and this was precipitated by adding methanol $(500 \mu \mathrm{l})$ and vortex-mixing for $1 \mathrm{~min}$. The mixture was centrifuged for $10 \mathrm{~min}$ at $1500 \times \mathrm{g}$, and the supernatant was transferred into a stoppered glass test tube. This was then evaporated to dryness under a nitrogen stream at $60^{\circ} \mathrm{C}$. The residue was trimethylsilylated by mixing with $50 \mu \mathrm{l}$ of BSTFA (containing $1 \% \mathrm{TMCS}$ ) and $50 \mu \mathrm{l}$ of acetonitrile, followed by incubation at $60^{\circ} \mathrm{C}$ for $20 \mathrm{~min}$. An aliquot of $2 \mu \mathrm{l}$ was injected onto a GC-MS instrument.

Instrumentation — A Shimadzu GCMS-QP2010 gas chromatograph mass spectrometer (Kyoto, Ja- 
pan) was operated in the positive electron ionization mode utilizing a DB-17 fused-silica capillary column $(30 \mathrm{~m} \times 0.32 \mathrm{~mm}$ i.d., film thickness $0.25 \mu \mathrm{m}$; J \& W Scientific, Folsom, CA, U.S.A.). Injections were made automatically in the splitless mode at an injection port temperature of $250^{\circ} \mathrm{C}$. Carrier gas was high-purity helium at a flow rate of $3.0 \mathrm{ml} / \mathrm{min}$. The column temperature was initially held at $60^{\circ} \mathrm{C}$ for $3 \mathrm{~min}$, and increased to $120^{\circ} \mathrm{C}$ at $10^{\circ} \mathrm{C} / \mathrm{min}$, then increased to $300^{\circ} \mathrm{C}$ at $30^{\circ} \mathrm{C} / \mathrm{min}$, and finally held at $300^{\circ} \mathrm{C}$ for $2 \mathrm{~min}$. The ionization energy and interface temperature were set at $70 \mathrm{eV}$ and $250^{\circ} \mathrm{C}$, respectively. Qualitative analysis was primarily carried out in the full-scan mode, but selectedion monitoring (SIM) mode was employed for a GHB level of $0.03 \mu \mathrm{g} / \mathrm{ml}$ or lower. Quantitative analysis was performed by the standard addition method, employing HCA as the IS. Two target ions $(\mathrm{m} / z 233$ for GHB and $\mathrm{m} / z 159$ for IS) and the following qualifier ions were monitored in the SIM mode: $\mathrm{m} / z, 147$ and 117 for GHB, and $m / z, 233$ and 147 for IS

\section{RESULTS}

\section{Procedure Optimization}

In order to accurately determine the low-nanogram-per-milliliter-level endogenous GHB in blood, a highly sensitive yet specific procedure should primarily be established. Several solid-phase extraction, liquid-liquid extraction, and protein precipitation methods were compared, by analyzing a fortified whole blood sample with GHB at $1.0 \mu \mathrm{g} / \mathrm{ml}$, for the recovery effectiveness, chromatographic cleanliness, and operation expediency. Liquid-liquid extraction gave a maximum recovery of $37 \%$ when ethyl acetate was used and the $\mathrm{pH}$ was adjusted to 4.4 with saturated ammonium chloride solution. The protein precipitation method provided the highest recovery of $63 \%$, while requiring no tedious sample handling and $\mathrm{pH}$ adjustment. It also provided chromatograms with acceptable cleanliness, as will be shown below. None of common-type solid-phase extraction cartridges tested retained GHB efficiently. Although the mixed-mode column CREEN SCREEN GHB provided a fair recovery of $57 \%$, the protein precipitation method provided somewhat clearer chromatograms than the other methods, which was advantageous for analyzing whole blood samples. By the use of BSTFA containing $1 \%$ TMCS, the derivatization of GHB and HCA (IS)

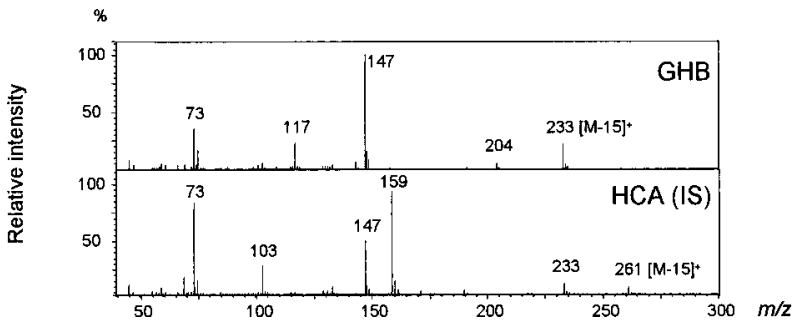

Fig. 2. Electron Impact Mass Spectra of the TMS Derivatives of GHB and HCA (IS)

(A)

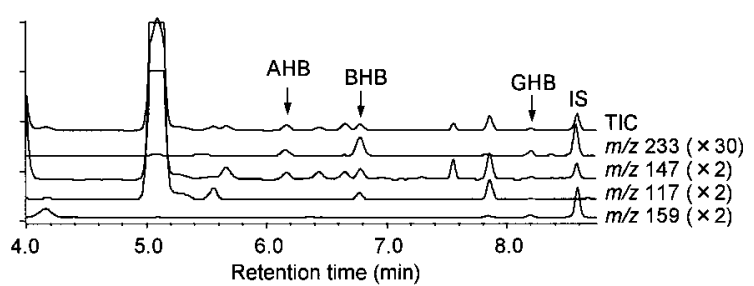

(B)

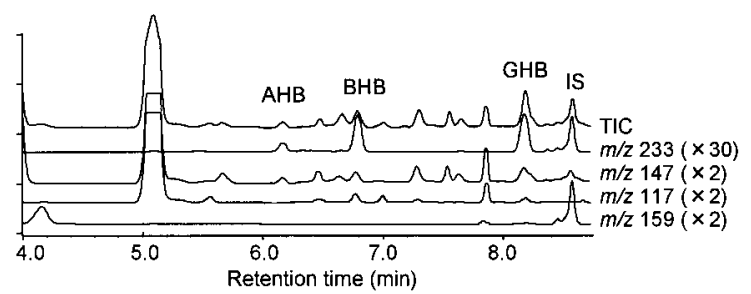

Fig. 3. Mass Chromatograms Obtained from (A) a Drug-Free whole Blood Sample and (B) that Spiked with GHB at $0.1 \mu \mathrm{g} / \mathrm{ml}$

even in whole blood matrix reached plateaus within $15 \mathrm{~min}$ at $60^{\circ} \mathrm{C}$, but a more prolonged incubation resulted in a colored sample mixture. Di-TMS derivatives were exclusively produced after a reaction time of $20 \mathrm{~min}$ for the target analyte and IS. Figure 2 shows the mass spectra of the derivatives of GHB and IS. The use of a medium polar DB-17 column provided satisfactory peak separations of GHB from its isomers, $\mathrm{AHB}$ and $\mathrm{BHB}$, as well as from blood components on the mass chromatograms of the monitoring ions at $m / e 233,117$, and 147 , under the GC conditions described in the experimental section. Figure 3 shows the mass chromatographic data obtained from a drug-free whole blood sample and a spiked whole blood sample with GHB at $0.1 \mu \mathrm{g} / \mathrm{ml}$.

Method Validation: The optimized procedure was validated by analyzing drug-free whole blood samples fortified with GHB and diluted standard solutions of GHB at known concentrations. The limits of detection (LODs), which was estimated from the detection limits of the target and qualifier ion peaks on each mass chromatogram $(\mathrm{S} / \mathrm{N} \geq 3)$, and 
satisfying the acceptable range of the ratio of qualifier to target ion responses $( \pm 20 \%)$, were estimated from the data of a drug-free whole blood sample and those of appropriately diluted standard solutions. The LODs were estimated to be 0.03 and $0.003 \mu \mathrm{g} / \mathrm{ml}$ in the full-scan and SIM modes, respectively. The recovery (\%) evaluated using a spiked blood sample at $1.0 \mu \mathrm{g} / \mathrm{ml}$ was $63 \%$. The recovery was calculated by comparing the peak area of the analyte extracted from a drug-free blood sample before and after spiking with GHB at $1.0 \mu \mathrm{g} / \mathrm{ml}$ and that from a $1.0 \mu \mathrm{g} / \mathrm{ml}$ diluted standard solution. Linearity ranged from 0.003 to $3.0 \mu \mathrm{g} / \mathrm{ml}$ with the correlation coefficiency of 0.999 . Accuracy evaluated at $0.1 \mu \mathrm{g} / \mathrm{ml}$ was 0.096 $\pm 0.015 \mu \mathrm{g} / \mathrm{ml}$ (average \pm S.D., $n=5$ ). Within-day and between-day precisions were 5.3 and $6.1 \%$, respectively $(n=5$ each). Thus, the validation data guaranteed the sensitivity and quantitative efficiency of the procedure.

\section{Endogenous Blood GHB Levels in Healthy Hu- mans}

In order to survey the endogenous level of GHB in blood, whole blood samples aseptically collected from 24 healthy volunteers were analyzed using the optimized procedure. The samples collected aseptically were processed and analyzed promptly, as detailed in the experimental section. The endogenous GHB levels ranged from 0.005 to $0.010 \mu \mathrm{g} / \mathrm{ml}$ with the average of $0.0069 \mu \mathrm{g} / \mathrm{ml}$ and the standard deviation of $0.0020 \mu \mathrm{g} / \mathrm{ml}$. The averages for males and females were $0.0067 \mu \mathrm{g} / \mathrm{ml}$ and $0.0072 \mu \mathrm{g} / \mathrm{ml}$, respectively. No obvious difference was noticed between male and female subjects.

\section{In Vitro GHB Production in whole Blood Samples}

Influence of Anticoagulants: Most blood samples submitted for drug testing are preserved by adding an anticoagulant. However, several papers ${ }^{13,14)}$ have suggested significant in-vitro production of GHB, especially in whole blood samples preserved with sodium citrate. ${ }^{13-15)}$ Thus, to investigate the influence of anticoagulants, the following commonly-used anticoagulants, each at a commonly-used concentration indicated in the parentheses, were mixed into freshly collected whole blood samples ( $n=3$ each), and the time course changes in GHB concentration were examined at $4^{\circ} \mathrm{C}$ : sodium citrate $(0.4 \%)$, EDTA $(0.1 \%)$, heparin $(0.001 \%)$, and sodium oxalate $(0.2 \%)$. Figure 4 presents the results.

The drug-free, in life whole blood sample used

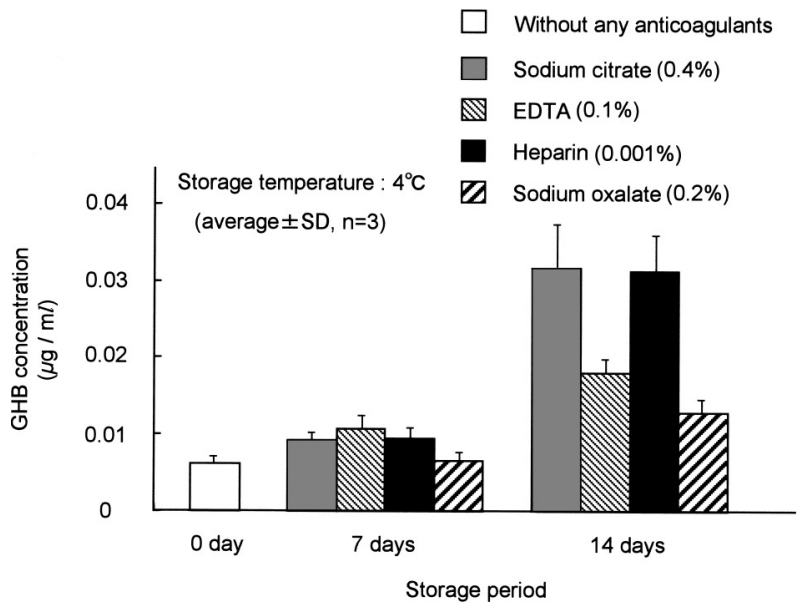

Fig. 4. Increase in the GHB Levels in Drug-Free whole Blood Samples Preserved with Commonly-Used Anticoagulants and Preservatives

for this experiment originally contained $0.006 \mu \mathrm{g} /$ $\mathrm{ml}$ of GHB. More than five times higher concentrations were detected in samples preserved with sodium citrate and heparin, but no noticeable effects were observed for sodium oxalate and EDTA.

Short-Term Storage Conditions: The in vitro GHB production was presumed to be due to the bacterial, enzymatic, or spontaneous decomposition of bioorganic blood components. Among these, bacterial decomposition was presumed to be the primarily source of in vitro GHB. Thus, the effects of sodium azide, one of the most popular preservatives, and storage temperature were examined for a storage period of two weeks. EDTA, which showed less effect on the GHB level, was also mixed at $0.1 \%$ as an anticoagulant instead of sodium oxalate because this organic acid may produce similar effect to sodium citrate. Figure 5 shows the results. The control whole blood sample used for this experiment originally contained $0.008 \mu \mathrm{g} / \mathrm{ml}$ of GHB. No notable increases were observed when sodium azide was used and stored at $4^{\circ} \mathrm{C}$, though an obvious increase in the GHB level was detected for the sample stored at room temperature. Thus, although the use of sodium citrate or heparin must be avoided, in-life whole blood samples collected aseptically can be stored for up to two weeks at $4^{\circ} \mathrm{C}$, when EDTA and sodium azide are added at $0.1 \%$ each.

Long-Term Storage Conditions: As a subject item of forensic investigation, biological samples often need to be stored for long periods of time. Thus, there is a requirement for investigating appropriate 


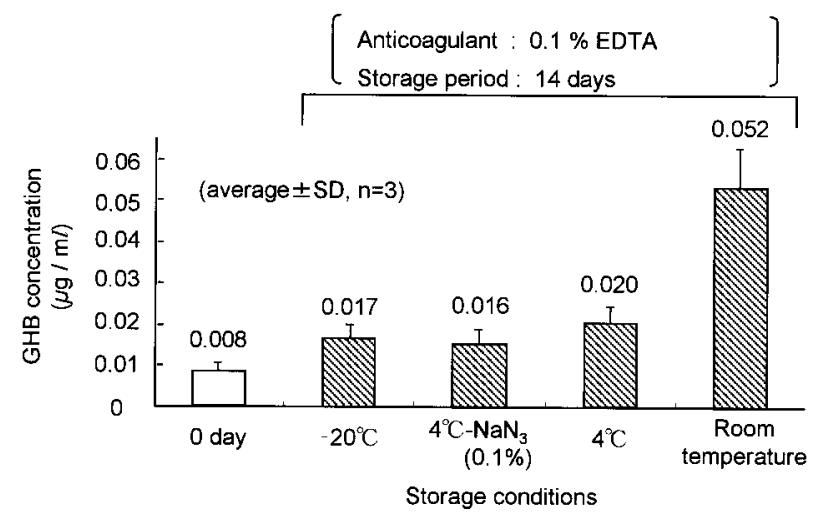

Fig. 5. Increases in the Blood GHB Concentration under Various Storage Conditions

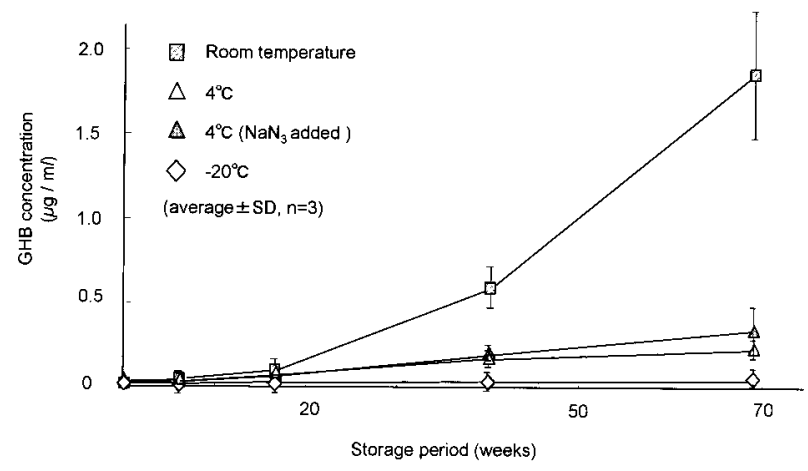

Fig. 6. Time Changes in the GHB Concentrations in whole Blood Samples Collected Aseptically from Three Healthy Volunteers, under Various Storage Conditions

storage conditions of whole blood samples, with special attention to the in-vitro production of GHB. Also, the level of GHB produced during the storage period should be investigated for appropriate interpretation of analytical results of GHB in blood samples. In this section, the effect of sodium azide was further examined for longer periods of storage. This experiment was carried out by using control blood samples aseptically collected from three healthy volunteers (31- and 50-year-old males, and 42 -year-old female). Figure 6 depicts the results. Substantial increases were observed for the storage at room temperature, while in vitro GHB production was less than 0.03 and $0.4 \mu \mathrm{g} / \mathrm{ml}$ at -20 and $4^{\circ} \mathrm{C}$, respectively, even after a storage period of 16 months. No remarkable effects were noticed for the addition of sodium azide in $4^{\circ} \mathrm{C}$ storage. Thus, blood samples should be frozen at $-20^{\circ} \mathrm{C}$, even for in-life samples collected aseptically, when the sample is to be stored for more than two weeks. However, the levels of in vitro GHB production in antemortem blood samples were found to be far below those in postmortem samples reported previously. ${ }^{7,8,13-15)}$

\section{DISCUSSION}

\section{Establishment of Procedure}

As described above, the protein precipitation method provided the highest recovery of $63 \%$, while requiring no tedious sample handling, including strict $\mathrm{pH}$ adjustment because blood is biologically buffered to $\mathrm{pH}$ 7.3-7.5. In extracting the acidic substance GHB by liquid-liquid extraction, the $\mathrm{pH}$ should be adjusted to 4.4 , usually with saturated ammonium chloride solution. However, the tautomeric conversion of GHB into GBL, which promptly occurs under acidic conditions, should carefully be avoided. In fact, Elliot demonstrated that analytical procedures involving liquid-liquid extraction tended to give lower GHB levels (mentioned by Elliot as "absolute GHB") than those involving the conversion of GHB into GBL with a strong acid and the detection as $\mathrm{GBL}^{7,16)}$ (the sum of GHB and GBL; mentioned by Elliot as "total GBL"). ${ }^{8)}$ Also, GHB does not have significant protein-binding properties. ${ }^{17)}$ These facts most probably contributed to the highest recovery of GHB in whole blood by the protein precipitation method adopted in the present study. In the present method, thus, GHB was extracted at an original $\mathrm{pH}$ of around 7.4, and was promptly trimethylsilylated to prevent such conversion to GBL. Owing to these facts, the present method provided satisfactory sensitivity in quantifying the low nanogram-per-milliliter level GHB in blood.

\section{Endogenous Blood GHB Levels}

The levels of GHB obtained in casework examination require critical interpretation, not only because it is a naturally-occurring substance in the body, but also because of the possible in-vitro production of GHB in biological samples. However, very little research has been undertaken to obtain trustworthy data for the endogenous level and the in-vitro production of GHB in blood from drug-free living humans, though this is essential in evaluating such levels of GHB in connection with criminal cases.

Elliott analyzed 15 serum samples submitted for toxicological examination and reported that no measurable GHB (less than $2.5 \mu \mathrm{g} / \mathrm{ml}$ ) was detected. ${ }^{18)}$ Elian analyzed 240 antemortem blood samples from 
presumably drug- and GHB-free subjects and reported that the endogenous blood GHB ranged from 0.17 to $1.51 \mu \mathrm{g} / \mathrm{ml}$, with a mean level of $0.74 \mu \mathrm{g} /$ $\mathrm{ml} .{ }^{19)}$ In our preliminary experiment, however, a prompt trace-level in vitro GHB production was regularly observed for freshly- but not asepticallycollected whole blood samples, even after storage for one night at $4^{\circ} \mathrm{C}$. Thus, in the present study, whole blood samples collected aseptically were analyzed promptly. As a result, the GHB levels measured ranged far below the "endogenous" range previously reported. The obvious difference from the above mentioned Elian's results ${ }^{19)}$ is probably attributed primarily to the in-vitro production of GHB before analysis (which will be discussed below) and differences in analytical techniques adopted in both studies, though differences in the nature of subject population, and eating and drinking tendencies must be included.

\section{Differences between Postmortem and Antemor- tem Blood Samples}

For postmortem blood samples, there has been increasing data demonstrating significant amounts of in-vitro production of GHB. ${ }^{7,8,14,15)}$ Fieler et al. documented that GHB was artificially elevated up to $168 \mu \mathrm{g} / \mathrm{ml}$ in 15 of 20 autopsy blood samples, ${ }^{7)}$ which overlaps the concentrations reported to be associated with its fatal reactions. Sakurada et al. analyzed five postmortem blood specimens from nonGHB cases and reported that the endogenous levels were $<0.4-2.6 \mu \mathrm{g} / \mathrm{g} .{ }^{20)}$ More recently, Moriya et al. detected 1.33-44.3 $\mu \mathrm{g} / \mathrm{ml}$ of GHB (average $9.8 \mu \mathrm{g}$ / $\mathrm{ml}$ ) in 40 out of 43 postmortem blood samples tested. ${ }^{15)}$ They also demonstrated that a large part of the GHB detected is generated during the interval between death and autopsy.

Generally, in-life blood samples were aseptically collected by using a sterilized disposable syringe and a reduced-pressure test tube. In these cases, blood samples, which are originally bacteria-free in the body, can be collected aseptically. On the contrary, blood in a corpse begins degenerating immediately after death, and thus, postmortem blood samples are usually contaminated with bacteria, often seriously. Also, it is well-established that the putrefaction of bioorganic substances yields a series of hydroxybutyric acids, including GHB. Thus, the much higher "endogenous" GHB levels in postmortem blood samples can be attributed to the bacterial production of GHB, as Moriya et al. also suggested. ${ }^{15)}$

Therefore, even though postmortem samples were cooled or frozen immediately after collection, contamination of such samples with bacteria and invitro-produced GHB is unavoidable and irremovable. In those cases, sodium azide, which inhibits bacterial activities, should be added, because such samples may be subjected to freezing and thawing cycles when used for multiple analyses. However, as shown in Fig. 6, no remarkable effects of sodium azide were shown for in-life blood samples that were collected aseptically. This implies that the bacterial in-vitro production of GHB makes no notable contribution to such in-life samples.

In terms of the effect of sodium citrate, significantly high levels of GHB have been frequently observed in postmortem blood preserved with sodium citrate. ${ }^{13,14)}$ Elliot reported GHB concentrations up to $197 \mu \mathrm{g} / \mathrm{ml}$ in postmortem blood samples preserved with sodium citrate. ${ }^{14)}$ To the contrary, in our separate experiment, a GHB level of $0.22 \mu \mathrm{g} / \mathrm{ml}$ was detected in an in-life whole blood sample that was collected aseptically and preserved by adding sodium citrate, even after a two-year storage at $4^{\circ} \mathrm{C}$. This level is far below those in postmortem samples preserved with sodium citrate. Although the exact mechanism of GHB production has not been identified, the bacterial production of GHB from citrate would primarily contribute to such high levels of GHB. Thus, postmortem and antemortem cases should be considered separately in the interpretation of GHB levels in the blood.

\section{Interpretation of GHB Levels in Antemortem Blood Samples}

There is a rare genetic disorder called GHB aciduria, which results from a deficiency of succinic semialdehyde dehydrogenase, producing an excessive accumulation of GHB. The endogenous GHB level in the blood of GHB acidurea patients reportedly reaches concentrations typically as high as $100 \mu \mathrm{g} / \mathrm{ml} .{ }^{21)}$ Thus, in proving GHB intake by blood testing, the possibility of GHB acidurea should not be excluded. The policy of the authors' laboratory suggests the collection of a subsequent blood sample to quantify the subject's basal level of GHB when legal evidence of GHB intake is required.

In terms of urine, the endogenous GHB level ranges between 0.16 and $3.03 \mu \mathrm{g} / \mathrm{ml},{ }^{12}$ and the levels of in vitro GHB production are usually comparable to or less than such endogenous levels. To the contrary, in terms of blood, the levels of in vitro GHB production are often much higher than those in inlife endogenous levels. Thus, another difficulty arises 
in the interpretation of blood GHB levels. Elian proposed a $5-\mu \mathrm{g} / \mathrm{ml}$ cutoff for the differentiation of exogenous from endogenous blood GHB. ${ }^{19)}$ The present research has been undertaken with special attention to the endogenous level and in-vitro production of GHB in blood aspiratory collected from living humans. The exogenous GHB level in blood reportedly increased to around $50 \mu \mathrm{g} / \mathrm{ml}$ after a typical oral dosage of $2.5 \mathrm{mg} / \mathrm{kg}$ GHB sodium salt. ${ }^{17)}$ Based on this information and the present research, the authors conclude that a cutoff of $5 \mu \mathrm{g} / \mathrm{ml}$ proposed by Elliott is generally acceptable for in-life whole blood samples stored at $4{ }^{\circ} \mathrm{C}$ or lower. However, in-life blood specimens are usually collected aseptically in Japan when it is executed based on a warrant for criminal investigation. Concerning such aseptic specimens, the maximum in-vitro GHB production observed during 14-day storage at $4^{\circ} \mathrm{C}$ was $0.03 \mathrm{ug} / \mathrm{ml}$, and the possible highest level is estimated to be $0.6 \mathrm{ug} / \mathrm{ml}$ when the safety margin is set at 20 . Also, the $99.99 \%$ upper confidence limit of the initial blood GHB level is calculated to be below $0.02 \mathrm{ug} / \mathrm{ml}$, based on the present data (average, $0.0069 \mathrm{ug} / \mathrm{ml}$; standard deviation, $0.0020 \mathrm{ug} / \mathrm{ml} ; n$ $=24)$. Thus, if only limited to such in-life blood specimens collected aseptically, an interpretative cutoff of $1.0 \mu \mathrm{g} / \mathrm{ml}$ would be appropriate for differentiating exogenous from endogenous GHB, when the sample is stored at $4^{\circ} \mathrm{C}$ or lower, examined within two weeks, and excludes the possibility of GHB aciduria.

\section{REFERENCES}

1) Nicholson, K. L. and Balster, R. L. (2001) GHB: a new and novel drug of abuse. Drug Alcohol Depend., 63, 1-22.

2) Feigenbaum, J. J. and Howard, S. G. (1996) Gamma hydroxybutyrate is not a GAVA agonist. Prog. Neurobiol., 50, 1-7.

3) Hornfeldt, C. H., Lothridge K. and Upshaw-Downs, J. C. (2002) Gamma-hydroxybutyrate (GHB). Microgram, 35, 102-113.

4) Fed. Regist., 65, 13235-13238 (2000).

5) Tunnicliff, G. (1997) Sites of action of gammahydroxybutyrate (GHB) - A neuroactive drug with abuse potential. Clin. Toxicol., 35, 581-590.

6) Doherty, J. D. and Roth, R. H. (1978) Metabolism of gamma-hydroxy- $\left[1-{ }^{14} \mathrm{C}\right]$ butyrate by rat brain: relationship to the Krebs cycle and metabolic compartmentation of amino acids. J. Neurochem.,
30, 1305-1309.

7) Fieler, E. L., Coleman, D. E. and Baselt R. C. (1998) GHB concentrations in pre and postmortem blood and urine (letter). Clin. Chem., 44, 692.

8) Elliott, S. P. (2004) Further evidence for the presence of GHB in postmortem biological fluid: Implications for the interpretation of findings. J. Anal. Toxicol., 28, 20-26.

9) Poldrugo, F. and Snead, O. C. (1984) 1,4-Butanediol, gamma-hydroxybutyric acid and ethanol: relationships and interactions, Neuropharmacology, 23, 109-113.

10) Schneir, A. B., Ly, B. T. and Clark, R. F. (2001) A case of withdrawal from the GHB precursors gamma-butyrolactone and 1,4-butanediol. J. Emerg. Med., 21, 31-33.

11) Rambourg-Schepens, M. O., Buffet, M., Durak, C. and Mathieu-Nolf, M. (1997) Gamma butyrolactone poisoning and its similarities to gamma hydroxyric acid: two case reports. Vet. Hum. Toxicol., 39, 234 235.

12) Shima, N., Miki, A., Kamata, T., Katagi, M. and Tsuchihashi, H. (2004) Urinary Endogenous Levels of GHB and Its Isomers in Healthy Humans and Diabetics. Forensic Sci. Int., in press.

13) LeBeau, M. A., Montgomery, M. A., Jufer, R. A. and Miller, M. L. (2000) Elevated GHB levels in citrate-buffered blood. J. Anal. Toxicol., 24, 383384.

14) Moriya, F. and Hashimoto, Y. (2004) Endogenous $\gamma$-Hydroxybutyric acid in postmortem blood. Leg. Med., 6, 47-51.

15) Elliot, S. P. (2001) The presence of GHB in postmortem biological fluids. J. Anal. Toxicol., 25, 152.

16) Stephens, R. G., Coleman, D. E. and Baselt, R. C. (1999) In vitro stability of GHB in postmortem blood. J. Forensic Sci., 44, 231.

17) Tedeschi, P. L., Frison, G., Padrini, R., Zordan, R., Orlando, R., Gallimberti, L., Gessa, G. L. and Ferrara, S. D. (1993) Dose-dependent absorption and elimination of gamma-hydroxybutyric acid in healthy volunteers. Eur. J. Clin. Pharmacol., 45, 353-356.

18) Elliott, S. P. (2003) Gamma hydroxybutyric acid (GHB) concentrations in humans and factors affecting endogenous production. Forensic Sci. Int., 133, 9-16.

19) Elian, A. A. (2002) Determination of endogenous gamma-hydroxybutyric acid (GHB) levels in antemortem urine and blood. Forensic Sci. Int., 128, 120 122.

20) Sakurada, K., Kobayasi, M., Iwase, H., Yoshino, M., Mukoyama, H., Takatori, T. and Yoshida, K. (2002) Production of gamma-hydroxybutyric acid in post- 
mortem liquid increases with time after death. Toxicol. Lett., 129, 207-217.

21) Jakobs, C., Kneer, J., Rating, D., Hanefeld, F., Divry,
P. and Hermier, M. (1984) 4-Hydroxybutyric aciduria: a new inborn error of metabolism. II. Biochemical findings. J. Inherit. Metab. Dis., 7, 92-94. 